\title{
Uber das Goursat-Problem für quasilineare hyperbolische Systeme partieller Differentialgleichungen erster Ordnung mit zwei unabhängigen Variablen
}

\author{
H.-P. GitTeL.
}

Die Arbeit behandelt die gemischte Anfangs-Randwertaufgabe für quasilineare hyperbolische Systeme erster Ordnung mit zwei Variablen. Zunächst wird untersucht, wieviel Randbedingungen man'stellen kann-und was die notwendigen Lösbarkeitsbedingungen (Kompatibilitätsbedingungen) sind. Als Vorbereitung zum Existenzbeweis in der Funktionenklasse $C^{k, 1}$ erfolgt weiterhin die Réduktion der Ausgangsaufgabe auf ein spezielles Goursat:Problem. Das zugehörige System (verlängertes System) hat,dann Diagonalgestalt, und es genügt; die Existenz Lipschitz-stetiger Lösungen für dieses Problem nachzuweisen.

В. работе изучается смешаниав задача для квазилинейных гиперболических систем первого порндка с двумл переменными. Исследуется, сколько монно поставить краевых

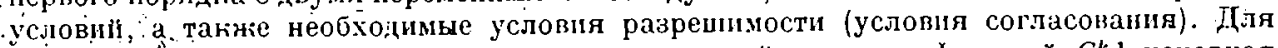
поцготовкй довазательстиа суцествования решениї в классе Функций $C^{k, 1}$ исходиая

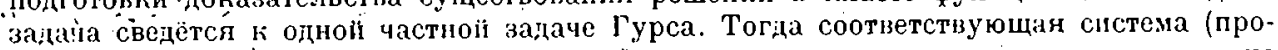
должениая система) имеет диагональную форму, и достаточно показать существование нёрерывных по Липиицу решений этой задачн.

$\because$.

This paper deals with the mixed problem for quasi-linear hyperbolic system of the first order in two variables At first we investigate how many boundary conditions may be set and what the necessary, conditions of solvability (compatibility conditions) are. In preparation for the existence proof in the function class $C^{k, 1}$ we reduce the original problem to a special Goursat problen. Then the corresponding system (prolonged system) has diagonal form and it is sufficient to prove the existence of Lipschitz continuous solutions for this problem.

\section{Einleitung}

Gegenstand der vorliegenden Arbeit ist: die gèmischte Anfangs-Randwertaufgabe, auch Goursat-Problem genannt, für das quasilineare hyperbolische System:

$$
\begin{aligned}
& \quad \sum_{j=1}^{m}\left(A^{i, j}\left(x, y, u_{i}^{1}, \ldots, u^{m}\right) \frac{\partial u^{j}}{\partial x}+B^{i, j}\left(x, y, u^{1}, \ldots, u^{m}\right) \frac{\partial u^{j}}{\partial y}\right) \\
& =F^{i}\left(x, y, u^{1}, \ldots, u^{m}\right) \quad(i=1, \ldots, m) . \\
& \text { Bei diesem } \\
& \text { Problem sind für eine Lösung } \mathbf{u}=\left(u^{i}\right)=\left(\begin{array}{c}
u^{1} \\
\vdots \\
u^{m}
\end{array}\right) \text { dieses Systems neben den }
\end{aligned}
$$
Anfangswerten auf einer (nichtcharakteristischen) Anfangskurve noch gewisse Rándbedingungen auf einer Randkurve vórgeschrieben. ${ }^{1}$ ) Spezielle Goursat-Probleme für (1.1) wurden von Prodse [13] und Thomée [15, 16] betrachtet, der lineare und

1) Andere Anfangs-Randwertprobleme für (1.1) wurden von BECKERT [1] behandelt. In [6: S. 30-33] wurde der Zusammenhang dieser Probleme mit der Aufgabenstellung des Goursat-

Problems untẹrsucht.

5 Analysis Bd. 4, Heft 1 (1085) 
semilineare Fall wurde z. B. in [4: S. 471-474] behandelt. Ziel der Untersuchungen ist neben der Analyse der zu stellenden Randrelationen und der notwendigen Lösbarkeitsbedingungen die Umformuing des Systems (1.1) auf' Diagonalform in 'das sogenannte verlängerte System. Dieses wurde von Covrant und LAX in [5] bei der. Betrachtung des üblichen Anfangswertproblems verwendet. Durch einige zusätzliche Schlußweisen und Herleitung geeigneter Randbedingungen gelingt es, ein zum Ausgangsproblem äquivalentes Goursat-Problem für das verlängerte System herzuleiten. Für dieses Problem kann dann mittels Differenzenverfahren ein Existenzbeweis erbracht werden, welchem eine weitere Abhandlung gewidmet ist [8].

Die vorliegende Arbeit ișt der erste Teil der Dissertation des Verfassers [7]. An - dieser Stelle möchte dieser besonders Prof. Dr. Beckert für die Anregung und Unterstützung dieser Untersuchungen darkèn.

Zunächst wollen wir folgende Festlegungen treffen:

Wir werden im weiteren die Räume $C^{k}(\bar{G})$ bzw. $C^{i, 1}(\bar{G})$ ( $k$ ganzzahlig nichtnegativ) verwenden. Dabei sei $G$ ein nichtleeres beschränktes Gebiet des $\mathrm{R}^{N}$. und $C^{r}(\bar{G})$ die Klasse aller reellwertigen Funktionen, die auf $\bar{G}$ stetige partielle Ableitungen bis zur Ordnung $k$ besitzen. $C^{k, 1}(\bar{G})$ sind alle Funktionen aus $C^{k}(\bar{G})$, deren höchste Ableitungen noch einer Lipschilz-Bedingung genügen ( $L$-stetig sind). Für q-dimensionale Funktionsvektoren $\mathbf{f}=\left(f^{h}\right)$ schreiben wir

$$
\left.\mathbf{f} \in C_{q}^{k}(\bar{G}) \text { und }\|\mathbf{f}\|_{C^{k}(\bar{G})}=\underset{h=1, \ldots, q}{\max }\left\|f^{h}\right\|_{C^{k}(\bar{G})}{ }^{2}\right) \text {, }
$$

falls für alle Komponenten $f^{h} \in C^{k}(\bar{G})(h=1, \ldots, q)$ gilt. Analoge Bezeichnungsweise gilt für die Räume $C_{q}^{k, 1}(G)$ und für Matrizen. Differentiation und Integration ist stets komponentenweise zu verstehen.

\section{Fin physikalisehes Beispiel}

Das bekannteste und Standard-Beispiel fïr unsere Aufgabenstellung ist der eindimensionale Gasstoß (Kolbenproblem).

Wir betrachten die eindimensionale Strömung eines Gases in einer Röhre, die in $\dot{x}$-Richtung ausgedehnt und an einem Ende mit einem Kolben verschlossen ist. Dieser wird mit vorgegebener Geschwindigkeit $v_{K}(t)$ in das Gas hineingeschoben oder
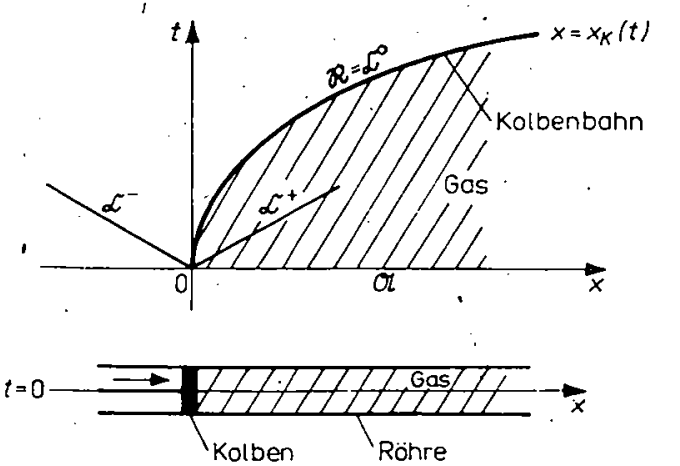

Abb. 1

$\left.{ }^{2}\right)\left\|f^{h}\right\| C^{k}(\bar{G})$ ist die übliche Maximumnorm in den Räumen $C^{k}(\bar{G})$ mit der Abkürzung $\left\|f^{h}\right\|_{\bar{G}}$ $:=\| f^{h} \mid c^{0}(\bar{G})$. 
herausgezogen (s. Abb. 1). Das Verhalten des Gases wird durch die eindimensionalen nichtisentropischen Strömungsgleichungen in Eulerscher Form besçhrieben [3:S. 28]:

$$
\left.\begin{array}{l}
\frac{\partial \varrho}{\partial \imath}+v \frac{\partial \varrho}{\partial x}+\varrho \frac{\partial v}{\partial x}=0 \\
\varrho \frac{\partial v}{\partial t}+\varrho v \frac{\partial v}{\partial x}+\frac{\partial p}{\partial x}=0 \\
\frac{\partial S}{\partial t}+v \frac{\partial S}{\partial x}=0,
\end{array}\right\},
$$

wobei die drei Unbekannten $v, \varrho, S$ nur von $x$ und der Zeit $t$ abhängen und $v$ die Gasgeschwindigkeit, $\varrho$ die Dichte und $S$ die spezifische Entropie bedeuten. Der Druck $p$ ist durch die Zustandsgleichung des Gases $p=Z(\varrho, S)$ gegeben.

Als Anfangskurve $\mathfrak{A}$ ist die $x$-Achse zu betrachten, da die Anfangswerte für $v, \varrho, S$ durch den Zustand des Gases zum Zeitpunkt $t=0$ festgelegt sind. Die Randbedingung ist durch die vorgeschriebene Gasgeschwindigkeit auf der Kolbenbahn $\left(x_{K}(t), t\right)$ für $t \geqq 0$. mit $x_{K}(0) \stackrel{=0}{=} \frac{d x_{K}}{d t}=v_{K}(t)$ 'in der $(x, t)$-Ebene gegeben :

$$
v\left(x_{K}(t), t\right)=v_{K^{\prime}}(t)
$$

so daß die Kolbenbahn die Randkurve $\mathfrak{\Re}$ darstellt.

Andere Beispiele fuir gemischte Anfangs-Randwertaufgaben findet man in $[2,6$ : S. $45-49,7$ : S. $13-16]$.

\section{Die charakteristische Form von (1.1)}

Für die weitere Behandlung von (1.1) ist es günstig, dieses System in Matrixschreibweise

$$
\mathbf{A} \frac{\partial \mathbf{u}}{\partial x}+\mathbf{B} \frac{\partial \mathbf{u}}{\partial y}=\mathbf{F}
$$

mit den Koeffizientenmatrizen $\mathbf{A}=\left(A^{i, j}\right), \mathbf{B}=\left(B^{i, j}\right)$ und dem Funktionenvektor $\mathbf{F}=\left(F^{i}\right)$ zu verwenden

Setzen wir Det $\mathbf{B} \neq 0$ voraus, so sind die Charakteristiken des Systems (3.1) diejenigen regulären Kurven $\mathfrak{C}$, für deren Richtungselement $\zeta=\frac{d x}{d y}$ gilt $(\mathbf{I}-m \times m-$
Einheitsmatrix)

$$
\therefore \quad \operatorname{Det}\left(\mathbf{B}^{-1} \mathbf{A}-\zeta \mathbf{I}\right)=0
$$

Es gibt $m$ Scharen von charakteristischen Kurven, jeweils mit der entsprechenden Vielfachheit der zugehörigen Wurzel von.(3.2) gezählt. Im hyperbolischen Fall, den wir hier ausschließlich betrachten, sollen sämtliche charakteristischen Richtungselemente $\zeta$ reell sein für alle in Frage kommenden Werte von $x, y, \mathbf{u}$. Die Voraussetzung Det $\mathbf{B} \neq 0$ gewährleistet auch, daß (3.1) nach $\frac{\partial \mathbf{u}}{\partial y}$ aufgelöst werden kann, und
weiterhin, daß die $x$-Achse keine Charakteristik ist.

Bemerkung 1: Wir werden o. B. d. A. annehmen, daß die Anfangskurve $\mathfrak{A}$ auf den Abschnitt [0,1] der $x$-Achṣe fällt, so daß $\mathfrak{A}$ wie beim Cauchy-Problem in keinem Punkt charakteristisch ist. Die Randkurve $\mathfrak{\Re}$. soll dabei der Abschnitt $[0,1$.$] der y$ - 
Achse sein ( $\lambda>0$, noch frei wählbarer Parameter). Diese Lage von Anfangs- und Randkurve kann durch eine geeignete Koordinatentransformation erreicht werden (s. z. B. [6: S. 12-19]).

Mit einer regulären Transformationsmatrix $\mathbf{T}=\left(T^{i j}\right)$ läßt $\operatorname{sich} \mathbf{B}^{-1} \mathbf{A}$ in die Weierstraß-Jordansche Normalform .überführen:

$$
\mathbf{T B}^{-1} \mathbf{A} \mathbf{T}^{-1}=\mathbf{D} \text {. }
$$

Wenn wir nun für das Weitere stets voraussetzen, da $B$ die Elementarteiler von $\mathbf{B}^{-1} \mathbf{A}$ - einfach, d. h. linear sind, hat $\mathbf{D}$ reine Diagonalgestalt:

$$
\mathbf{D}=\left(\begin{array}{ccc}
D^{1} & \ldots & 0 \\
\vdots & \ddots & \vdots \\
0 & \ldots & D^{n}
\end{array}\right)
$$

Thre Diagonalelemente $D^{i}=D^{i}(x, y, \mathbf{u})$ sind die $m$ Wurzeln der Gleichung (3.2). Sie sollen so angeordnet sein, daß gilt:

$$
D^{i} \leqq 0 \quad \text { für } i=1, \ldots, r, \quad D^{l}>0 \quad \text { für } l=r+1, \ldots, m .
$$

Unter diesen Voraussetzungen an $\mathbf{B}^{-1} \mathbf{A}$ und $\mathbf{D}$ erhalten wir mit (3.3) das zu (3.1). $\therefore$ äquivalente System, die charakteristische Form:

$$
\mathbf{T} \frac{\partial \mathbf{u}}{\partial y}+\mathbf{D}^{\prime} \mathbf{T} \frac{\partial \mathbf{u}}{\partial x}=\mathbf{T B}^{-1} \mathbf{F}=: \overline{\mathbf{F}} .
$$

Bemerkung 2: Die Voraussetzung, daß die Elementarteiler der Matrix $\mathbf{B}^{-1} \mathbf{A}$ einfach sein sollen, ist insbesondere dann gewährleistet, wenn alle $m$ Wurzeln der charakteristischen Gleichung (3.2) \% $\mathbf{B ~}^{-1} \mathrm{~A}$ verschieden sind (total hyperbolischer Fall). Dieselbe Voraussetzung an die Elementarteiler von $\mathbf{B}^{-1} \mathbf{A}$ findet man auch bei der Behandlung anderer. Anfangs-Randwertprobleme z. B. in [1] wieder. Diese Bedingung berücksichtigt auch die Tatsache, daß der Fall nicht einfacher Elementarteiler Systeme mit einschlie Bt, für die das Anfangswertproblem keine Iösung besitzt [12: S. 551$]$.

Bemerkung 3: Die in (3.4) geforderte Anordnung kann bei $D^{i} \neq 0$ stets durch entsprechende Zeilenvertauschung im Systèm (3.5), bei eventueller Einschränkung des gemeinsamen Definitionsbereiches der $D^{i}$, erreicht werden, wenn wir deren vorausgesetzte Stetigkeit ausnutzen. Nur im Fall $D^{i}(x, 0, \mathbf{u}(x, 0))=0$ muB Näheres über das Verhalten von $D^{i}$ in einer Umgebung von $(x, 0, \mathbf{u}(x, 0))$ bekannt sein (s. auch Bemerkung 4).

\section{Die Randbedingungen}

Ist $\mathfrak{S}_{i}$ die Charakteristik, die von $\mathfrak{O}=(0,0)$ ausgeht und das Richtungselement $\frac{d x}{d y}=D^{i}$ hat, so verlaufen die $(m-r)$ Charakteristiken $\mathfrak{S}_{r+1}, \ldots, C_{m}$ innerhalb eines Winkelbereichs zwischen $x$ - und $y$-Achse, die restlichen $r$ Stück $\mathfrak{V}_{1}, \ldots, \mathfrak{C}_{r}$ außerhalb davon, jeweils mit ihrer Vielfachheit gezählt. Dabei können die $\mathfrak{C}_{i}(i=1, \ldots, r)$ die $\grave{y}$-Achse in einigen Punkten berühren, da $D^{i}(0, y, u(0, y))=0$ zugelassen ist, oder auch einige der $\mathfrak{S}_{i}$ mit der $y$-Achse zusammenfallen (s. Abb. 2). Solche Fälle konnten sowohl von THomée $[15,16]$ als auch von Prouse [13] nicht bericksichtigt werden. Jedoch ändern sich die Verhältnisse für die Lösbarkeit, insbesondere die Anza,hl der 
zu stellenden Randbedingungen, nicht, wenn $\Re$ charakteristisch ist, wie man bei der Konstruktion einer Lösung für das Goursat-Problem aus dén Lösungen anderer Anfangs- und Randwertprobleme nach BEckert [1] erkennt [6: S. 30-33]. Dabei werden genau so viele Randbedingungen, nämlich $(\dot{m}-r)$, gestellt, wie es Charakteristiken gibt, die von $\mathfrak{D}$ ausgehend in einem Winkelbereich zwischen $\mathfrak{A}$ und $\mathfrak{A}$ verlaufen. Allerdings mußten bei dieser Konstruktion einige der Randbedingungen auf diesen Charakteristiken gestellt werden.

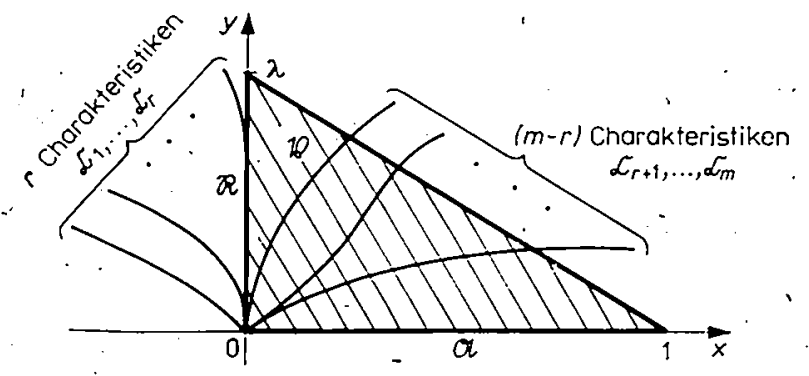

Abb. 2

Unser Problèm lautet nun folgendermaßen:

P I : Gesucht wird eine Lösung $\mathbf{u}$ von (3.5) in

$$
\therefore \quad . \dot{\mathcal{B}}=\left\{(x, y) \in \mathrm{R}^{2} \mid x \geqq 0, y \geqq 0, \lambda x+y \leqq \lambda\right\}
$$

mit

und

$$
u^{i}(x, 0)=g^{i}(x) \quad \text { für } x \in[0,1], \quad i=1, \ldots, m .
$$

$$
\sum_{j=1}^{m} R^{\prime . j}(y, \mathbf{u}(0, y)) \frac{\partial u^{j}}{\partial y}(0, y)=H^{\prime}(y ; \mathbf{u}(0, y))
$$

für $y \in[0, \lambda], \quad l=r+1, \ldots, m$.

Wir schreiben hier also die $(m-r)$ Randbedingungen (4.2) auf der gegebenen Randkurve $\Re$ vor. Diese sollen linear unabhängig sein, $d . h$. die Zeilen der Randmalrix $\mathbf{R}=\left(R^{l \cdot j}\right)$ sind für alle $(y, \mathbf{u}) \in \bar{Q}^{0}$ linear unabhängig. Mit $\mathbf{R}$ und $\mathbf{H}=\left(H^{\prime}\right)$ schreiben wir (4.2) auch in der Form:

$$
\mathbf{R} \frac{\partial \mathbf{u}}{\partial y}=\mathbf{H} \quad \text { für } \quad x=0 .
$$

Weiterhin stellen wir folgende Voraussetzungen:

V I : $\quad \cdot \quad \mathbf{T}, \mathbf{D} \in C_{m \times m}^{k, 1}(\bar{Q}), \quad \overline{\mathbf{F}} \in C_{m}^{k, 1}(\bar{Q})$,

mit

$$
\mathbf{R} \in C_{(m-r) \times m}^{k-1,1}\left(\bar{Q}^{0}\right), \quad \mathbf{H} \in C_{m-1}^{k-1,1}\left(\bar{Q}^{0}\right), \quad g \in C_{m}^{k, 1}([0,1])
$$

und

$$
\left.\bar{Q}=\left\{\left(x, y, u^{1}, \ldots, u^{m}\right) \in \mathrm{R}^{2+m}|0 \leqq x \leqq 1 ; 0 \leqq y \leqq \sigma,| \mathbf{u}-g(x) \mid \leqq \varrho\right\}^{3}\right)
$$$$
\bar{Q}^{0}=\left\{\left(y, u^{1}, \ldots, u^{m}\right) \in \mathbf{R}^{1+m}|0 \leqq y \leqq \sigma,| \mathbf{u}-\mathbf{g}(0) \mid \leqq \varrho\right\},
$$

$\left(k \_\geqq 1\right.$, ganzzahlig; $\sigma, \varrho>0$ reell).

3) Für $z \in R^{N}$ sei $|z|=\underset{v=1 \ldots \ldots, N}{\max }\left|z^{\nu}\right|$. 
Bemerkung 4: Betrachten wir die lineare partielle Differentialgleichung erster Ordnung $u_{y}+x u_{x}=0$, so ist $D \equiv x>0$ fiir $x \in(0,1]$. Man könnte deshalb annehmen, daß es wie im Fall $D(x, y, u)>0$ auf $\bar{Q}$ möglich wäre, für $u$ auf $\Re$ noch eine Randbedingung vorzuschreiben. Das ist jedöch hier nicht möglich, denn die Charakteristik durch $\mathfrak{D}$ ist die Gerade $x=0$, und $u(0, y)$ ist schon durch die Anfangswerte bestimmt. Auf Grund dieser Verhältnisse zwischen Nichtnegativität von $D$ und Randbedingungen haben wir die Fälle $D^{l} \geqq 0$ für $l=r+1, \ldots, \dot{m}$ aus unseren Betrachtungen ausgeschlossen (s. (3.4)).

Kehren wir noch einmal zu unserem physikalischen Beispiel (Kolbenproblem) zuriick, so ist festzustellen, daß dabei die Verhältnisse genau so vorliegen, wie wir es in unserer allgemeinen Problemstellung P I annehmen. Wir haben für (2.1) folgende 3 Ćharakteristiken:

$$
\begin{aligned}
& \mathfrak{C} \pm: \frac{d x}{d t}=v \pm c, \text { zwei Schallwellen, } \\
& \mathfrak{c}^{0}: \frac{d x}{d t}=v ; \text { Teilchenbahn, }
\end{aligned}
$$

wobei $c$ die Schallgeschwindigkeit bedeutet $\left(c^{2}=\frac{\partial p}{\partial \varrho}\right)$. Die von $\bigcirc$ ausgchende Schallwelle $\mathfrak{S}^{+}$. verläuft zwischen positiver $x$-Achse und Kolbenbahn (s. Abb. 1), und es war gerade eine Randbedingung (2.2) gestellt worden. $Z u$ benierken ist noch, da $\beta$ in diesem Beispiel die Randkurve - die Kolbenbahn - gerade charakteristisch ist (bei isentropischer Strömung nicht).

\section{Kompatibilitätsbedingungen}

Durch Ưbertragung der in Thomée [15, 16] und in Krsyzanski und Schá́der [10] angeführten Schlußweisen ergeben sich Kompatibilitätsbedingungen für die Anfangswerte (4.1) und die Randbedingungen (4.2) als notwendige Lösbarkeitsbedingungen.

Satz 1: Ist P I unter den Voraussetzungen V I in der Klasse $C_{m}^{1}(\mathfrak{B})$ lösbar, so müssen die Kompatibilitätsbedingungen erster Ordnung

$$
\begin{aligned}
& \mathbf{R}(0, \mathbf{g}(0)) \mathbf{T}^{-1}(0,0, \mathbf{g}(0)) \\
& \left(\overline{\mathbf{F}}(0,0, \mathbf{g}(0))-\mathbf{D}(0,0, \mathbf{g}(0)) \mathbf{T}(0,0, \mathbf{g}(0)) \mathbf{g}^{\prime}(0)\right)=\dot{\mathbf{H}}(0, \mathbf{g}(0))
\end{aligned}
$$

erfïllt sein.

Beweis: Differentiation von (4.1) liefert

$$
\frac{\partial \mathbf{u}}{\partial x}(x, 0)=\frac{d \mathbf{g}}{d x}(x) \text {. }
$$

Betrachten wir (3.5) speziell in $\mathfrak{D}$, so erhalten wir

$\frac{\partial^{\prime} \mathbf{u}}{\partial y}(0,0)=\mathbf{T}^{-1}(0,0, \mathbf{g}(0))\left(\overline{\mathbf{F}}(\mathbf{0}, 0, g(0))-\mathbf{D}(0,0, \mathbf{g}(0)) \mathbf{T}(0,0, \mathbf{g}(0)) \mathbf{g}^{\prime}(0)\right)$.

Einsetzen dieser Beziehung in (4.2) für $y=0$ liefert (5.1)

Satz 2: Hat P I unter den Voraussetzungen $\mathbf{V}$ I eine Lösung in $C_{m}^{k}(\mathfrak{B})$, so sind die Komputibilitütsbedingungen erster bis $k$-ter Ordnung erfüllt. 
Bemerkung 5. Wir beweisen im folgenden nưr die Behauptung des Satzes für $k=2$ (für $k=1$. s. Satz 1), wobei klar ersichtlich wird, wie der. Beweis im Fall $k>2$ verläuft. Dadurch können wir auch auf die explizite Angabe aller der recht umfangreichen Kompatibilitätsbedingungen bis zur Ordnung $k$ verzichten.

Beweis von Satz 2: a) Ist $\mathbf{u} \in C_{m}^{2}(\mathfrak{V})$ eine Lösung von $\mathbf{P} \mathbf{I}$, so gelten nach dem Beweis von Satz 1 die Beziehungen (5.1) bis (5.3).

b) Um die Kompatibilitätsbedingungen zweiter Ordnung herzuleiten; intersuchen wir die zweiten Ableitungen von, $u$ in $\mathscr{D}$ und bilden dann mittels (3.5) und (4.2) solche Bèziehungen, die nur noch die gegebenen Ainfangswerte und Koeffizienten sowie deren Ableitungen in $\mathfrak{D}$ enthalten.

b 1) Differentiation von (4.2) bez. $y$ liefert

$$
\left.\frac{d}{d y} \mathbf{R}\right|_{\mathbf{0}} \frac{\partial \mathbf{u}}{\partial y}(0,0)+\left.\mathbf{R}\right|_{0} \frac{\partial^{2} \mathbf{u}}{\partial y}(0,0)=\left.\frac{d}{d y} \mathbf{H}\right|_{\mathbf{0}}
$$

Für eine Funktion $Z(x, y, \mathbf{u})$ bedeutet:

$$
\begin{aligned}
& Z_{10}=Z(0,0, \dot{g}(0)) \text {, } \\
& \frac{d}{d y} Z_{10}=\frac{\partial Z}{\partial y}(0,0, \mathbf{g}(0))+\sum_{j=1}^{m} \frac{\partial Z}{\partial u^{j}}(0,0, \mathbf{g}(0)) \frac{\partial u^{j}}{\partial y}(0,0) \\
& =\frac{\partial Z}{\partial y}{ }_{10}+\left(\left.\operatorname{grad}_{\mathbf{u}} Z\right|_{0}\right)^{T} \mathbf{T}_{10}^{-1}\left(\overline{\mathbf{F}}_{l_{0}}-\left.(\mathbf{D T})\right|_{0} \mathbf{g}^{\prime}(0)\right) \text { wegen }(5.3) \text {, } \\
& \left.\frac{d}{d x} Z\right|_{0}=\frac{\partial Z}{\partial \dot{x}}(0,0, \mathbf{g}(0))+\sum_{j=1}^{n} \frac{\partial Z}{\partial u^{j}}(0,0, \mathbf{g}(0)) \frac{\partial u^{j}}{\partial x}(0,0) \text {. } \\
& =\frac{\partial Z}{\partial x} \mathrm{i}_{0}+\left(\left.\operatorname{grad}_{\mathbf{u}} Z\right|_{0}\right)^{T} \mathbf{g}^{\prime}(0) \quad \text { wegen (5.2). }
\end{aligned}
$$

b2) Um $\frac{\partial^{2} u^{\circ}}{\partial y}(0,0)$ aus (5.4) zu èliminieren, wird das.System (3.5) bez. $y$ differenziert und nach dieser Größe aufgelöst:

$\frac{\partial^{2} \mathbf{u}}{\partial y}(0,0)=, \mathbf{T}_{10}^{-1}\left(\frac{d}{d y} \overline{\mathbf{F}}_{10}-\frac{d}{d y}(\mathbf{D T})_{10} \mathbf{g}^{\prime}(0)-(\mathbf{D T})_{10} \frac{\partial^{2} \mathbf{u}}{\partial y \partial x}(0,0)-\frac{d}{d y} \mathbf{T}_{0} \frac{\partial \mathbf{u}}{\partial y} \cdot(0,0)\right)$.

Wegen $\frac{\partial^{2} \mathbf{u}}{\partial y \partial x}=\frac{\partial^{2} \mathbf{u}}{\partial x \partial y}$ ergibt sich in $\mathfrak{D}$ die Größe $\frac{\partial^{2} \mathbf{u}}{\partial y \partial x}$ a us dem nach $x$ differenzierten System (3.5):

$\frac{\partial^{2} \mathbf{u}}{\partial x \partial \dot{y}}(0,0)=\mathbf{T}_{10}^{-1}\left(\frac{d}{d \mathbf{x}} \cdot \mathbf{F}_{10}-\frac{d}{d x}(\mathbf{D T})_{\left.\right|_{0}} \mathbf{g}^{\prime}(0)-(\mathbf{D T})_{10} \cdot \mathbf{g}^{\prime \prime}(0) \cdot-\frac{d}{d x} \mathbf{T}_{10} \frac{\partial \mathbf{u}}{\partial y}(0,0)\right)$.

ь3) Einsetzen dieser beiden letzten Gleichungen und der Beziehung (5.3) in (5.4) liefert die Kompatibilitätsbedingungen zweiter Ordnung

$$
\begin{aligned}
& \mathbf{R}_{\mid 0} \mathbf{T}_{10}^{-1}\left(\frac{d}{d y} \overline{\mathbf{F}}_{10}-\mathbf{D}_{10} \frac{d}{d x} \overline{\mathbf{F}}_{10}-\left(\frac{d}{d y}(\mathbf{D T})_{10}-D_{10} \frac{d}{d x}(\mathbf{D T})_{10}\right) \mathbf{g}^{\prime}(0)\right. \\
& \left.+\left(\mathbf{D}^{2} \mathbf{T}\right)_{10} \mathbf{g}^{\prime \prime}(0)-\left(\frac{d}{d y} \mathbf{T}_{10}-\mathbf{D}_{10} \frac{d}{d x} \mathbf{T} \mathbf{T}_{0 \mid}\right) \mathbf{c}\right)=\frac{d}{d y} \mathbf{H}_{10}-\frac{d}{d y} \mathbf{R}_{10}
\end{aligned}
$$

mit $\mathrm{e}=\mathrm{T}_{10}^{-1} \cdot\left(\overline{\mathbf{F}}_{10}-(\mathbf{D T})_{10} \mathbf{g}^{\prime}(0)\right)$ 
Sind nur $(q-r)$ linear unabhängige Randbedingungen der Form (4.2). gegeben $(r \leqq q \cdot<m)$ und $(m-q)$ implizite Randrelationen, d. h. gilt für $y \in[0, \lambda]$

$$
\sum_{j=1}^{m} R^{l, j}(y, \mathbf{u}(0, y)) \frac{\partial u^{j}}{\partial y}(0, y)=H^{\prime}(y, \mathbf{u}(0, y)), \quad l=r+1, \ldots, q .
$$

und

$$
\hat{H}^{\prime}(y, \mathrm{u}(0, y))=0, \quad l_{1}=q+1, \ldots, m,
$$

so läßt sich dieser Fall in unsere weitéren Betrachtungen einordnen $\div$ man erhält daraus Randbedingungen vom Typ (4.2), wenn (5.7) bei $\left.\hat{\mathbf{H}} \in C_{m-q}^{\mathbf{1}}\left(\bar{Q}^{0}\right), u \in C_{m}^{1}(\hat{\mathcal{Q}}\}\right)$ bez. $y$ differenziert wird und falls die Zeilen der neuen. Randmatrix linear unabhängig sind für alle $(y, u) \in \bar{Q}^{0}$. Liegen Randbedingungen vom Typ (5.6); (5.7) vor, so folgt - aús der Stetigkeit von $\mathbf{u}$ in $\mathfrak{D}$ der

Satz 3: Gibt es eine Lösung $\mathbf{u} \in C_{m}^{1}(\mathfrak{B})$ von (3.5), (4.1), (5.6), (5.7), so genügen $\hat{\mathbf{H}}$ und g. zusätzlich den Kompatibilitätsbedingungen nullter Ordnung

$$
\hat{H}^{\prime}(0, \mathbf{g}(0))=0, \quad l=\dot{q}+1, \ldots, m .
$$

Bemerkung 6: Die Bedingungen (5.8) sichern für u $\in C_{m}^{1}(\mathfrak{Z})$ mit den Anfangswerten (4.1) gerade die Äquivalenz zwischen (5.7) und den sich aus (5.7) ergebenden differenzierten Relationen, so da $\beta$ wir uns im weiteren auf Randbedingungen vom Typ (4.2) beschränken können.

Wir haben bis jetzt die Notwendigkeit der Konpatibilitätsbedingungen für die Lösbarkeit von Goursat-Problemen zu (3.5) gezeigt. Bei einigen Anfangs-Randwertproblemen zu einfachen partiellen Differentialgleichungen erster Ordnung kann man unmittelbar nachrechnen; daß diese Aufgabenstellungen bei $k$-mal stetig differenzierbaren Koeffizienten, Anfangs- und Randwerten génau dann eine Lösung aus $C^{k}([0,1] \times[0,1])$ besitzen; falls die Kompatibilitätsbedingungen bis zur Ordnung $k$ erfüllt sind [7: S. 40-42]. Für den allgemeinen Fall quasilinearer Systeme (3.5) wird die Hinlänglichkeit dieser Bedingungen in einer folgenden Arbeit [8] untersucht. Dazu ist erst eine weitere geeignete Transformation von (3.5) erforderlich.

\section{UUb erfïhrung quasilinearer hyperbolischer Systeme in Diagonalform}

Fs erweist sich als günstig, System (3.5) in Diagonalform zu bringen, weil sich dadurch das Differenzenverfahren für den Existenzbeweis sowie die notwendigen Abschät.zungen vereinfachen; d. h., wir wollen (3.5) in.ein System des Aussehens

$$
\frac{\partial \tilde{\mathbf{u}}}{\partial y}+\ddot{\mathbf{D}}(x, y, \tilde{\mathbf{u}}) \frac{\partial \tilde{\mathbf{u}}}{\partial x}=\tilde{\mathbf{F}}(x, y, \tilde{\mathbf{u}})
$$

'umformen. Für lineare und semilineare Systeme.(3.5), also solche Systeme,.in denen $\mathbf{T}$ und $\mathbf{D}$ nicht von $\mathbf{u}$ abhängen, ist das in bekannter. Weisè möglich, indem neue Unbekannte $\overline{\mathbf{u}}=$ Tu eingeführt werden. Für den quasilinearen Fall ist eine solche Einführung neuer Unbekannter jedoch nicht geeignet. Courasi und Lax haben in [5] ein Verfähren vorgeschlagen, mit dèm für quasilineare hyperbolische Systeme (3.5) durch Einführung von zusätzlichen Unbekannten ein Diagonalsystem hergeleitet wird. Wir werden im folgenden eine geringe Modifizierung dieses Verfa'hrens' auf unser Goursat-Problem anwenden und untersuchen, wie die Randbedingungè dabei zu berïcksichtigen sind. Dabei betrachten wir besonders den Fall, wenn eine Lösung u von (3:5) nur $L$-stetige Ableitungen besitzt. 
Wir nehmen an, daß u eine Lösung des Systems (3.5) sei, die in einem gewissen Teilbereich

$$
\mathfrak{B}^{2}=\left\{(x, y) \in \mathrm{R}^{2} \mid x \geqq 0,0 \leqq y \leqq \tau \lambda, i x+y \leqq \lambda\right\}, \quad 0<\tau \leqq 1,
$$

des Winkelbereichs $\mathfrak{B}$ definiert ist. Für die Koeffizienten voñ (3.5) soll gelten: $T^{i, j}$, $D^{i}, \vec{F}: \in C^{1,1}(Q)$ für $i, j=1, \ldots, m$. Diese Funktionen sind dann auch aus der Klasse $C^{0,1}(\bar{Q})$, denn das Gebiet $Q=\operatorname{int} \bar{Q} \subset \mathrm{R}^{2+m}$ erfüllt die Bedingung $(S)^{4}$ ) (zum Beweis s. [7: S. 45-47]). Von $u \in C_{m}^{1.1}\left(\mathfrak{B}^{\mathrm{r}}\right)$ fordern wir $\left.\|u-g\|_{\mathfrak{B}_{\mathrm{r}}} \leqq \varrho^{5}\right)$ für hinreichend kleines $\tau$. Wählen wir $\tau \leqq \frac{\sigma}{\lambda}$, dann gelten die Inklusionen $\left.T^{i, j}[\mathbf{u}], D^{i}[\mathbf{u}], \bar{F}^{i}[\mathbf{u}] \in C^{1,1}\left(\mathfrak{Q}^{1}\right) .{ }^{\mathbf{r}}\right)$ Jetzt führen wir zusätzlich die neuen Funktionen $v^{1}, \ldots, v^{m}, w^{1}, \ldots, w^{m}$ durch die . Definitionen

$$
\mathbf{v}:=\mathbf{T} \frac{\partial \mathbf{u}}{\partial x} \quad \text { und } \quad \mathbf{w}:=\mathbf{T} \frac{\partial \mathbf{u}}{\partial y}
$$

ein. Mit diesen Größen wird (3.5) zu

$$
\mathbf{w}+\mathbf{D v}=\overline{\mathbf{F}}
$$

Wegen v, w $\in C_{m}^{0.1}\left(\mathfrak{Z}^{\mathrm{r}}\right)$ können wir auf Grund des Satzes von Rademacher [14] die letzte Gleichung bez. $x$ differenzieren und erhalten so folgendes Differentialgleichungssystem f. ü. in, $\mathfrak{Z}^{\mathrm{r}}$ :

$$
\frac{\partial \mathbf{w}}{\partial x}+\mathbf{D} \frac{\dot{\partial} \mathbf{v}}{\partial x}=\frac{d}{d x} \overline{\mathbf{F}}-\frac{d}{d x} \mathbf{D} \mathbf{v}
$$

Da die partiellen Ableitungen der $L$-stetigen Funktionen $\frac{\partial u^{i}}{\partial x}, \frac{\partial u^{i}}{\partial y}$ f. ü. mit dèn entsṕrecheriden Distributionsableitungen zusammenfallen ${ }^{\natural}[11:$ S. 273-274]; gilt für. f. a. $(x, y) \in \mathfrak{B}^{2}$

$$
\text { , : } \frac{\partial}{\partial y}\left(\frac{\partial u^{i}}{\partial x}\right) \fallingdotseq \frac{\partial}{\partial x}\left(\frac{\partial u^{i}}{\partial y}\right)
$$
Auf Grund der entsprechenden Ausdrücke (aus (6.2)) läßt $\operatorname{sich} \frac{\partial w}{\partial x} \operatorname{durch} \frac{\partial v}{\partial y}$ mit Hilfe
von (6.5) f. ü. in $\mathfrak{B}^{2}$ darstellen, so daß aus (6.4) folgt:

$$
\frac{\partial \mathbf{v}}{\partial y}+\mathbf{D} \frac{\partial \mathbf{v}}{\partial x}=\frac{d}{d x} \overline{\mathbf{F}}-\frac{d}{d x} \mathbf{D} \mathbf{v}+\frac{d}{d y}, \mathbf{T} \frac{\partial \mathbf{u}}{\partial x}-\frac{d}{\partial x} \mathbf{T} \frac{\partial \mathbf{u}}{\partial y}
$$

. Erșetzen wir in der rechten Seite dieses Systems die partiellen Ableitungen von u gemäß (6.2) durch $\frac{\partial \mathbf{u}}{\partial x}=\mathbf{N v}, \frac{\partial \mathbf{u}}{\partial y}=\mathbf{N w}, \mathbf{N}=\mathbf{T}^{-1}$, so erhalten wir dafür einen bekann-

-) Die Bedingung (S) wurde in [11 : S. 24] definiert und sichert die Einbettung $C^{k+1}(\bar{G}) \subset C^{k, 1}(\bar{G})$, falls $G$ dieser Bedingung genügt. Dies gilt insbesondere für konvexe und sternförmige Gebiete. 5) Vergleiche FuBrote auf Seite 66.

6) Für cine Funktion $Z$, definiert auf $\vec{Q}$, wird zur Abkürzung $Z[\mathbf{u}]:=Z(\cdot, \cdot, \mathbf{u}(\cdot, \cdot))$ gesetzt. 
ten Ausdruck $\mathbf{F}_{*}=\mathbf{F}_{*}(x, y, \mathrm{u}, \mathrm{v}, \mathbf{w})$ mit

$$
\begin{aligned}
F_{*}{ }^{i}= & \frac{\partial \bar{F}^{i}}{\partial x}+\left(\operatorname{grad}_{\mathrm{u}} \bar{F}^{i}\right)^{T} \cdot \mathbf{N v}-\left(\frac{\partial D^{i}}{\partial x}+\left(\operatorname{grad}_{\mathrm{u}} D^{i}\right)^{T} \cdot \mathbf{N v}\right) v^{i} \\
& +\sum_{j=1}^{m}\left(\frac{\partial T^{i, j}}{\partial y}+\left(\operatorname{grad}_{\mathrm{u}} T^{i, j}\right)^{T} \cdot \mathbf{N w}\right) \sum_{h=1}^{m^{\prime}} N^{i, h} v^{h} \\
& -\sum_{j=1}^{m}\left(\frac{\partial T^{i, j}}{\partial x}+\left(\operatorname{grad}_{\mathrm{u}} T^{i, j}\right)^{T} \cdot \mathbf{N v}\right) \sum_{h=1}^{m} N^{j, h} w^{h}
\end{aligned}
$$

ganzrational in jeder der Variablen $v^{1}, \ldots, v^{m}, w^{1}, \ldots, w^{m}$, und $L$-stétig bez. $(x, y, \mathrm{u}$, $\mathbf{v}, \mathbf{w}) \in \bar{Q} \times\left[-\varrho_{1}, \varrho_{1}\right]^{m} \times\left[-\varrho_{2}, \varrho_{2}\right]^{m} \subset R^{2+3 m}$. mit beliebigen $\varrho_{1}, \varrho_{2}>0$. Wir haben damit aus (3.5) für die $3 m$ Komponenten von $u, v$, w ein System von $3 m$ G Gleichungen hergeleitet, nämlich (6.3), (6.6) und

$$
\frac{\partial \mathbf{u}}{\partial y}=\mathbf{N} \mathbf{w}
$$

(6.3) gestattet die Elimination von w, und wir erhalten endgültig ein geeignetes System von $2 m$ Differentialgleichungen in Diagonalform, das sogenannte verliingerte System zu (3.5):

$$
\begin{aligned}
& \frac{\partial \mathbf{u}}{\partial y}=\mathbf{N}(\overline{\mathbf{F}}-\mathbf{D} \mathbf{v}) \\
& \left.\frac{\partial \mathbf{v}}{\partial y}+\mathbf{D} \frac{\partial \mathbf{v}}{\partial x}=\overline{\mathbf{F}}_{*} \quad \text { (f. ii. in } \mathfrak{B}^{r}\right)
\end{aligned}
$$

${ }_{v} \operatorname{mit} \overline{\mathbf{F}}_{*}=\overline{\mathbf{F}}_{*}(x, y, \mathbf{u}, \mathbf{v})=\mathbf{F}(x, y, \mathbf{u}, \mathbf{v}, \overline{\mathbf{F}}(x, y, \mathbf{u})-\mathbf{D}(x, y, \mathbf{u}) \mathbf{v})$.

Bemerkung 7: Bei einer Herleitung gemäß [5] muß man die Gleichungen (6.3) bez. $y$ differenzieren. Man erhält daraus ein analoges System (6.9), jedoch für u und $\mathrm{w}$, falls $D^{i} \neq 0$ für $i=1, \ldots, m$ ist, was in unserem Fall aber nicht vorausgesetzt. wurde.

Wir haben somit den folgenden Satz bewiesen (der Fall $k>1$ ist offensichtlich).

Satz 4: Jeder Lösung u $\in C_{m}^{k .1}\left(\mathfrak{B}^{2}\right)$ von (3.5) mit Koeffizienten aus $C^{k, 1}(\bar{Q})$ entspricht eine Lösung $\tilde{\mathbf{u}} \doteq\left(\begin{array}{c}\mathbf{u} \\ \mathbf{v}\end{array}\right) \in C_{2 m}^{k-1.1}\left(\mathfrak{B}^{\mathbf{r}}\right)$ von $(6.9)$. mit Koeffizienten aus
$C^{k-1,1}\left(\bar{Q} \times \cdot\left[-o_{1}, o_{1}\right]^{m}\right)$.

Besonders günstig für unsere weiteren Betrachtungen ist, daß sich die Charakteristiken beim Ubergang vom System (3.5) zum verlängerten System (6.9) nicht wesentlich ändern. Gegenübèr (3.5) kommen für (6.9) als charakteristische Scharen, nur die Geraden $\frac{d x}{d y} \equiv 0$ hinzu, und zwar $m$-mal gezählt. Danit wird für (6.9) éntweder die $y$-Achse zusätzlich charakteristisch oder ihre Vielfach heit als Charakteristik erhöht sich bez. (3.5) um $m$. Von entscheidender Bedeutung ist, daß sich dic AnzahI der Charakteristiken mit pośitivem Anstieg beim Úbergang vón (3.5) zu (6.9) nicht ändert. Damit können für die Lösungen beider Systeme die gleiche Anzahl von Randbedingungen gestellt werden (s. Abschnitt 4). Welche Randbedingungen zu stellen sind, wird im Abschnitt 8 untersucht. 


\section{7. Äquivalenz zwisehen Ausgangssystem und transformiertem System in Diagonalform}

Bei der Existenztheorie [8] wollen wir uns auf die Betrachtung von einfacheren Systemen in Diagonalform (6.1) beschränken. Dazu ist noch der Nachweis notwendig, da $B$ jeder Lösung von (6.9) eine Lösung von (3.5) entspricht (Umkehrung des Satzes 4), womit dann die Äquivalenz dieser beiden Systeme vollständig bewiesen wäre. Dabei miissen besondere Schlußweisen herangezogen werden, falls $(6.9)$ nur $f$. ü. in $\mathfrak{B}^{r}$ von einem $L$-stetigen $\tilde{\mathbf{u}}$ - erfüllt wird. Dazu sind zunächst einige Betrachtungen über $L$ stetige Funktionen notwendig, die auf einem Rechteck $\Omega=(a, b) \times(c, d) \subset \mathrm{R}^{2}$ definiert sind und dort eine $L$-stetige partielle Ableitung besitzen.

Hilfssatz 1 : Sind f und $\frac{\partial f}{\partial y} L$-stetig auf $\bar{\Omega}$, dann ist

$$
\int_{a}^{x} \cdot\left(\int_{c}^{y} \frac{\partial}{\partial x}\left(\frac{\partial f}{\partial y}(\xi, \eta)\right) d \eta\right) d \xi=\int_{a}^{x}\left(\frac{\partial f}{\partial x}(\xi, y)-\frac{\partial f}{\partial x}(\xi, c)\right) d \xi
$$

fïr jedes $(x, y) \epsilon, \bar{\Omega}$.

Beweis: Für festes $y_{0} \in[c, d]$ ist $f\left(\cdot, y_{0}\right)$ absolut stetig auf $[a, b]$. Deshalb ergibt sich der Wert des Integrals auf der rechten Seite von (7.1) nach dem Satz von Lebesgue [9: S. 344] als

$$
f(x, y)-f(a, y)-(f(x, c)-f(a, c)) .
$$

Denselben Wèrt erhalten wir für die linke Seite von (7.1), wenn wir zunächst den Satz von Fubini über $[a, x] \times[c, y] \subseteq \bar{\Omega}$ anwenden und anschließend den Satz von Lebesgue auf $\frac{\partial f}{\partial y}(\cdot, \eta)$ für festes $\eta \in[c, y] \mathbf{~}$

Hilfssatz 2 : Sind $f, g$ L-stetig auf $\bar{\Omega} \cdot$ und $\frac{\partial f}{\partial x}=g f \cdot \ddot{u}$. in $\Omega$, so existiert $\frac{\partial f}{\partial x} \ddot{u}$ berall in $\Omega$ mit $\frac{\partial f}{\partial x} \equiv g$, also $\frac{\partial f}{\partial x} \in C^{0,1}(\bar{\Omega})$. (Eine analoge Aussage gilt fuir $\frac{\partial f}{\partial y}$.)

Beweis: Sei $x \in[a, b]$. Nach Integration von $\frac{\partial f}{\partial x}=g$ über $[a, x] \times[c, y] \subseteq \bar{\Omega}$ erhalten wir mit Anwendung des Satzes von Lebesgue

$$
\int_{c}^{\prime \prime}\left(\int_{a^{\prime}}^{x} g(\xi, \eta) d \xi\right) d \eta=\int_{c}^{\grave{v}}(f(x, \eta)-f(a, \eta)) d \eta \text { für alle } y \in[c, d]
$$

Dabei sind die Integrale auf beiden Seiten die entsprechenden Riemannschen Integrale wegen der Stetigkeit von $f$ und $g$. Differentiation dieser Beziehung bez. $y$ liefert für alle $(x, y) \in \Omega$ die Darstellung $\int_{a}^{x} g(\xi, y) d \xi=f(x, y)-f(a, y)$ und damit die Behauptung

Wie im , vorigen Abschnitt setzen wir T, $\mathbf{D} \in C_{m \times m}^{1,1}(\bar{Q}), \overline{\mathbf{F}} \in C_{m}^{1,1}(\bar{Q})$ voraus. Sei $\tilde{\mathbf{u}}=\left(\begin{array}{l}\mathbf{u} \\ \mathbf{v}\end{array}\right) \in C_{2 m}^{0,1}\left(\mathfrak{B}^{r}\right)$ eine Lösung von (6.9) f. ü. in $\mathfrak{B}^{r}$ mit $\|\mathbf{u}-\mathbf{g}\|_{\mathfrak{B}_{\mathfrak{r}}} \leqq \varrho,\|\mathbf{v}\|_{\mathfrak{B}_{\mathfrak{r}}} \leqq \ddot{\varrho_{1}}$. 
Dann liefern die ersten $m$ Gleichungen von (6.9)

$$
\mathbf{T} \frac{\partial \dot{\mathbf{u}}}{\partial y}+\overline{\mathbf{p}} \mathbf{v}=\overline{\mathbf{F}}
$$

$\mathbf{u}$ ist folglich dann eine I,ösung von (3.5), falls $\mathbf{v}=\mathbf{T} \frac{\partial \mathbf{u}}{\partial x}$ bez. $\frac{\partial \mathbf{u}}{\partial x}=$ Nv gilt. Wir betrachten dazu die Differenz. $\delta:=\mathbf{N} \mathbf{v}=\frac{\partial \mathbf{u}}{\partial x}$.

Hilfssatz 3 : Sind die Anfangswerte, so vorgegeben; daß $\delta(x, 0)=0$ für $x \in[0,1]$ gilt, dann ist unter den obigen Bedingungen $\delta \equiv 0$ in $\mathfrak{B}^{\circ}$ und $\mathbf{u}$ eine Lösung von (3.5) aus der. Klasse $C_{m}^{\mathrm{t}, 1}\left(\mathfrak{B}^{\mathrm{r}}\right)$.

Beweis: a) Wir wählen zu $x_{1} \in(0,1)$ ein Rechteck $\Omega_{1}=\left(0, x_{1}\right) \times\left(0, a\left(x_{1}\right)\right) \subset \mathfrak{B}^{\mathrm{r}}$ mit $\alpha(x)=\lambda \min \{\tau, 1-x\}$. Wegen $\mathbf{u}, \mathbf{v} \in C_{m}^{0,1}\left(\mathfrak{B}^{\tau}\right)$ ist die rechte Seite in den ersten $m$ Gleichungen von (6.9) $L$-stetig auf $\bar{\Omega}_{1}$ und Hilfssatz 2 ergibt $\frac{\partial \mathbf{u}}{\partial y} \in \bar{C}_{m}^{0,1}\left(\bar{\Omega}_{1}\right)$ sowie
die Gültigkeit von (7.2) auf ganz $\bar{\Omega}_{1}$.

b) Für den Nachweis $\delta \equiv 0$ auf $\bar{\Omega}_{1}$ leiten wir Integralgleichungen für die beschränkten, meßbaren Funktionen $\delta^{1}, \ldots, \delta^{m}$ her. ${ }^{7}$ ) b 1) Wegen der $L$-Stetigkeit von $N v$ und $\frac{\partial u}{\partial y}$ auf $\bar{\Omega}_{1}$ erhalten wir f. ï. in $\Omega_{1}$ aus den
letzten $m$ Gleichungen von (6.9)

$$
\frac{\partial(\mathbf{N v})}{\partial y}=\frac{d}{d y} \mathbf{N} \mathbf{v}+\mathbf{N} \frac{\partial \mathbf{v}}{\partial y}=\frac{d}{d y} \mathbf{N} \mathbf{T} \mathbf{N} \mathbf{v}+\mathbf{N}\left(\dot{\overline{\mathbf{F}}}_{*}-\mathbf{D} \frac{\partial \mathbf{v}}{\partial x}\right)
$$

und aus den ersten $m$ Gleichungen durch Differentiation bez. $x$

$$
\frac{\partial}{\partial x}\left(\frac{\partial \mathbf{u}}{\partial y}\right)=\frac{d}{d x} \mathbf{N}(\overline{\mathbf{F}}-\mathbf{D} \mathbf{v})+\mathbf{N}\left(\frac{d}{d x} \overline{\mathbf{F}}-\frac{d}{d x} \mathbf{D} \mathbf{v}-\mathbf{D} \frac{\partial \mathbf{v}}{\partial x}\right)
$$

Daraus folgt unter Verwendung der nach $y$ bzw. $x$ differenzierten Beziehung $\mathbf{N T}=\mathbf{I}$ :

$$
\frac{\partial(\mathbf{N} \hat{\mathbf{v}})}{\partial y}=\mathbf{N}\left(-\frac{d}{d y} \mathbf{T} \mathbf{N} \mathbf{v}+\overline{\mathbf{F}}_{*}+\frac{d}{d x} \mathbf{T} \frac{\partial \mathbf{u}}{\partial y} \cdot \frac{d}{d x} \overline{\mathbf{F}}+\frac{d}{d x} \mathbf{D} \mathbf{v}\right)+\frac{\partial}{\partial x}\left(\frac{\partial \mathbf{u}}{\partial y}\right) .
$$
Berücksichtigt man $\overline{\mathbf{F}}_{*}(x, y, \mathbf{u}, \mathbf{v})=\mathbf{F}_{*}\left(x, y, \mathbf{u}, \mathbf{v}, \mathbf{T} \frac{\partial \mathbf{u}}{d y}\right)$ sowie die Gestalt von $\mathbf{F}_{*}$
gemäß (6.7), so erhält man

$$
\frac{\partial(\mathbf{N v})}{\partial y}=\mathbf{P} \boldsymbol{\delta}+\frac{\partial}{\partial x}\left(\frac{\partial \mathbf{u}}{\partial y}\right)
$$

für f. a. $(x, y) \in \Omega_{1}$. Dabei besteht die Koeffizientenmatrix $\mathbf{P}=\left(P^{i, j}\right)$ für die gegebenen Funktionen $\mathbf{u}=\mathbf{i}(x, y), \mathbf{v}=\mathbf{v}(x, y)$ aus beschränkten, meßbaren Funktionen $P^{i, j}$ für $i, j=1, \ldots, m_{.}{ }^{7}$ )

- b2) Zu $y \stackrel{\prime}{\epsilon}\left[0, \alpha\left(x_{1}\right)\right]$ integrieren wir (7.3) über $[0, x] \times[0, y] \subseteq \Omega_{1}$ für $x \in\left[0, x_{1}\right]$ und erhalten mit Anwendung des Satzes von Lebesgue [9:S. 344] auf Nv sowie von

7) In den Punkten von $23^{x}$, wo diese Funktionen nicht definiert sind, also in einer $z$ weidimensionalen Nullmenge, werden sie 0 gesetzt. 
Formel (7.1) auf $\mathbf{u}, \frac{\partial u}{\partial y}$ :

$$
\begin{aligned}
\int_{0}^{x}(\mathbf{N v}(\xi, y)-\mathbf{N v}(\xi, 0)) d \xi= & \int_{0}^{x}\left(\int_{0}^{y} \frac{\partial(\mathbf{N v})}{\partial y} d \eta\right) d \xi \\
= & \int_{0}^{x} \cdot\left(\int_{0}^{y} \mathbf{P}(\xi, \eta) \delta(\xi, \eta) d \eta\right) d \xi \\
& +\int_{-}^{x}\left(\frac{\partial \mathbf{u}}{\partial x}(\xi, y)-\frac{\partial \mathbf{u}}{\partial x}(\xi, 0)\right) d \xi .
\end{aligned}
$$

Wegen der Definition von $\delta$ gilt dann

$\int_{0}^{x} \delta(\xi, y) d \xi=\int_{0}^{x} \delta(\xi, 0) d \xi+\int_{0}^{x}\left(\int_{0}^{\nu} \mathbf{P}(\xi, \eta) \delta(\xi, \eta) d \eta\right) d \xi \quad$ für alle $x \in\left[0, x_{1}\right]$

๖3) Für festes $y \in\left[0, \alpha\left(x_{1}\right)\right]$. können wir die letzte Gleichung bez. $x$ differenzieren,

- wobei wir $\int_{0}^{x} \ldots d \xi$ als unbestimmte Lebesguesche Integrale auffassen, und erhalten mit $[9:$ S. 336$]$.

$$
\boldsymbol{\delta}(x, y)=\boldsymbol{\delta}(x, 0)+\int_{0}^{\nu} \mathbf{P}(x, \eta) \boldsymbol{\delta}(x, \eta) d \eta
$$

für'f. a. $x \in\left[0, x_{1}\right]$.

c) Wir schätzen $\delta$ ab. (7.4) ist die gesuchte Integralgleichung für $\delta$, aus dēr wir von $\boldsymbol{\delta}(x, 0)=0$ für $x \in\left[0, x_{1}\right]$ auf $\delta \equiv 0$ in $\Omega_{1}$ schließen können. Setzen wir zur Abkürzung

$$
\begin{aligned}
& M=\max _{i, j=1, \ldots, m}\left(\sup _{(x, y) \in \mathfrak{B}_{r}}\left|P^{i, j}(x, y)\right|\right) \text { und } \chi^{i}(y)=\int_{0}^{y}\left(\int_{0}^{x_{1}}\left|\delta^{i}(\xi, \eta)\right| d \xi\right) d \eta \\
& (i=1, \ldots, m),
\end{aligned}
$$

so ist $\chi^{i}$ stetig auf $\left[0, \alpha\left(x_{1}\right)\right]$. Für $\chi(y)=\max _{i=1, \ldots, m} \chi^{i}(y)$ folgt aus (7.4) die Abschätzung:

$$
\chi(y) \leqq m M \int_{0}^{y} \not(\eta) d \eta \text { für alle } y \in\left[0, \alpha\left(x_{1}\right)\right]
$$

Nach dem Gronwallschen Lemma [17: S. 37] ist dann $\chi(y) \equiv 0$ auf $\left[0, \alpha\left(x_{1}\right)\right]$ und damit $\delta=0$ bzw. $\frac{\partial u}{\partial x}=\mathbf{N v}$ f. ü. auf $\Omega_{1}$. Wegen Hilfssatz 2 gelten die letzten beiden Gleichungen sogar iiberall auf $\Omega_{1}$.

d) Für $\mathfrak{B}^{r}$ gilt folgende Darstellung als Vereinigung der $\vec{\Omega}_{1}=\vec{\Omega}_{1}\left(x_{1}\right): \mathfrak{B}^{\mathrm{r}}=\underset{x_{1} \in(0.1)}{\cup} \vec{\Omega}_{1}\left(x_{1}\right)$ $-\cup\{(1,0)\} \cup\{(0, \tau \lambda)\}$. Da (7.2) (nach a)) und $\delta \equiv 0$ (nach c)) auf allen $\bar{\Omega}_{1}\left(x_{1}\right)$ gelten, erhalten wir die Behauptung 
Bemerkung 8: Für $\tilde{\mathbf{u}} \in C_{2 m}^{1}\left(\mathfrak{B}^{r}\right)$ vereinfacht sich der Beweis, da wir mit Hilfe des * Saties von Schwarz aus (7.3) ein lineares homogenes Differentialgleichungssystem für $\delta$ herleiten können:

$$
\frac{\partial \boldsymbol{\delta}}{\partial y}=\mathbf{P} \boldsymbol{\delta}
$$

mit stetigen Koeffizienten Pi.j. Nach bekannten Sätzen besitzt (7.5) dann nur die triviale Lösung bei $\delta(\dot{x}, 0)=0$ [5, 7:S. 52-53].

Für die Äquivalenz der Systeme (3.5) und (6.9) spielen wegen der Voraússetzung im letzten Hilfssatz die Anfangswerte von u und v cine entscheidende Rolle. Wenn u die Anfángswerte (4.1) anninmt, so hat $\tilde{\mathbf{u}}=\left(\begin{array}{l}\mathbf{u} \\ \mathbf{v}\end{array}\right)$ gemäß der Definition (6.2) von v die
Anfangswerte

$$
\ddot{\mathbf{u}}(x, \dot{0})=\left(\begin{array}{l}
\mathbf{g}(x) \\
\mathbf{T}(x, 0, \mathbf{g}(x)) \dot{\mathbf{g}}^{\prime}(x)
\end{array}\right)=: \tilde{\mathbf{g}}(x)
$$

auf $[0,1]$. Wir haben bei $\mathbf{g} \in \dot{C}_{m}^{k, 1}([0,1]) ; \mathbf{T} \in C_{m \times m}^{k .1}(\bar{Q})$ die Inklusion $\tilde{\mathbf{g}} \in C_{2 m}^{k-1, \dot{1}}([0,1])$. (7.6) sind nun gerade die richtigen Anfangswerte, unter denen die Äquivalenz von (3.5) und (6.9) wegen

$$
\delta(x, 0)=\mathbf{N}(x, 0, \mathbf{u}(x, 0)) \mathbf{v}(x, 0)-\frac{\partial \mathbf{u}}{\partial x}(x, 0)=\mathbf{0}
$$

gilt, so daß wir zusammenfassend den folgenden Satz formulieren können.

Satz 5: Sei V I erfüllt. Dann ist zu jeder Lösung $\tilde{\mathbf{u}}=\left(\begin{array}{l}\mathbf{u} \\ \mathbf{v}\end{array}\right) \in C_{2 m}^{k-1,1}\left(\dot{\mathfrak{B}} \xi^{\tau}\right)$ von $(\dot{6} .9)$, (7.6) u eine Lösung von (3.5), (4.1).aus der Klasse $C_{m}^{k .1}\left(\mathfrak{B}^{z}\right)$. (Für $k=1$ gilt (6.9) nur f. ü. in $\mathfrak{B}$.)

Wie wir sehen, hängt die Äquivalenz der Systeme (3.5) und (6.9) nur von den Anfangswerten $a b$, und die Randbedingungen haben keinen Einfluß auf dieses Problem. Es bleibt zu untersuchen, wie sich die Randbedingungen (4.2) für eine Lösung von (3.5). geeignet in den Unbekannten $u, v$ von (6.9) ausdrücken lassen.

\section{Randbedingungen für das Diagonalsystem}

Wir betrachten eine Lösung $u \in C_{m}^{1,1}\left(\mathfrak{B}^{\mathrm{r}}\right)$ von (3.5), die den Randbedingungen (4.2) genügt. Gemäß Satz 4 erfüllt unter den dort genıachten Voraussetzungen $\tilde{\mathbf{u}}=\left(\begin{array}{l}\mathbf{u} \\ \mathbf{v}\end{array}\right)$ das Differentialgleichungssystem (6.9) in $\mathfrak{B}^{\mathfrak{}}$. Die ersten $m$ Gleichungen von (6.9), speziell auf der Geraden $x=0$, geben uns die Möglichkeit, aus (4.2) sofort Randbedingungen herzuleiten, in denen keine Ableitungen von $\tilde{\mathbf{u}}$ auftreten: Da für $y \in[0, \tau i]$

$$
\frac{\partial \mathbf{u}}{\partial y}(0, y)=\mathbf{N}(0, y, \mathbf{u}(0, y))\langle\overline{\mathbf{F}}(0, y, \mathbf{u}(0, y))-\mathbf{D}(0, y, \mathbf{u}(0, y)) \mathbf{v}(0, y))
$$

gilt, ist nämlich (4.2) äquivalent zu

$$
\mathbf{R N}(\overline{\mathbf{F}}-\mathbf{D v})=\mathbf{H} \quad \text { für } \dot{x}=\mathbf{0} \text {. }
$$

Es bleibt die Frage, ob es möglich ist, (8.2) nach gewissen $v^{l}$ aufzulösen, so daß sich diese als Funktionen der restlichen $v^{i}$ und $\mathbf{u}$ darstellen lassen. Speziell wollen wir ex- 
plizite Randbedingungen für $v^{\tau+1}, \ldots, v^{m}$ erhalten. Das sind gerade diejenigen Komponenten von $\tilde{\mathbf{u}}=\left(\begin{array}{l}\mathbf{u} \\ \mathbf{v}\end{array}\right)$, für die in der zugehörigen Differentialgleichung von (6.9) das Diagonalelement $D^{l}$ positiv ist (s. Anordnung (3.4)).

Zunächst sind zur Behandlung dieser Frage die folgenden einfachen Betrachtungen über Matrizen und Vektoren notwendig.

Definition: Bezüglich (3.4) definieren wir für einen m-dimensionalen Vektor $\mathbf{z}$ die Vektoren

$$
\mathrm{z}^{-}=\left(\begin{array}{c}
z^{1} \\
\vdots \\
z^{\tau}
\end{array}\right) \quad \text { und } \quad \mathrm{z}^{+}=\left(\begin{array}{c}
z^{r+1} \\
\vdots \\
z^{m}
\end{array}\right)
$$

Für eine $m \times m$-Matrix $\mathbf{K}$ sei

$$
\mathbf{K}^{-}=\left(\begin{array}{ccc}
K^{1,1} & \ldots & K^{1, m} \\
\vdots & & \\
K^{r, 1} & \ldots & K^{r, m}
\end{array}\right) \text { und } \mathbf{K}^{+}=\left(\begin{array}{ccc}
K^{r+1,1} & \ldots & K^{r+1, m} \\
\vdots & & \ddots \\
K^{m, 1} & \ldots & K^{m, m}
\end{array}\right)^{?}
$$

Ist $\overline{\mathbf{K}}$ eine Diagonalmatrix, so schreiben wir zur Abkürzung

$$
\overline{\mathbf{K}}^{-}=\left(\begin{array}{ccc}
\bar{K}^{1} & \ldots & 0 \\
\vdots & \ddots & \vdots \\
0 & \ldots & \overline{\bar{K}}^{r}
\end{array}\right) \text { und }, \overline{\mathbf{K}}^{+} .=\left(\begin{array}{cccc}
\bar{K}^{r+1} & \ldots & 0 \\
\vdots & \ddots & \vdots \\
0 & \ldots & \bar{K}^{m}
\end{array}\right)
$$

Wir fordern nun für das. Weitere von den Randbedingungen (4.2), daß die Zeilen der Randmatrix $\mathbf{R}$ und die ersten $r$ Zeilen der Matrix $\mathbf{T}(0, y, \mathbf{u})$ insgesamt linear unabhängig sein sollen, n. a. W.: für die erweiterte Randmatrix

gelte

$$
\mathbf{S}=\mathbf{S}(y, \mathbf{u})=\left(\begin{array}{l}
\mathbf{T}^{-}(0, y, \mathbf{u}) \\
\mathbf{R}(y, \mathbf{u})
\end{array}\right)
$$

$$
\text { Det } \mathbf{S} \neq 0 \text { für alle }(y, \mathbf{u}) \in \overline{\mathbf{Q}}^{\mathbf{0}} \text {. }
$$

Bemerkung 9: Verzichtet man auf die Forderung. (8.3), so könnte mán stets eine Randbedingung angeben, für die die zugehörige erweiterte Randmatrix $\mathbf{S}$ singulär wird und unter bestimmten Umständen diese Relation keine zusätzliche Bedingung: an die Lösung von (3.5) darstellt [ $7:$ S. 63].

Åhnliche Determinantenbedingungen wie (8.3) findet man auch in $[1,13]$. Nur wenn man wíe Tноме́E in $[15,16]$ Systeme bereits in Diagonalform, betrachtet, kommt man auf Grund der speziellen Gestalt des Systems ohne solche Voraussetzungen an die Randbedingungen aus.

Unter der Bedingung (8.3) gilt der folgende Hilfssatz, aus dem wir dann den Satz über äquivalente Randbedingungen gewinnen. können.

Hilfssatz-4: Sei $\mathbf{R}=\dot{\mathbf{R}}(y, \mathbf{u})$ und $\mathbf{T}=\mathbf{T}(0, y, \mathbf{u})$ für festes $(y, \mathbf{u}) \in \bar{Q}^{\circ}$. Gilt.für zwei Vehtoren $\mathrm{z}=\left(\begin{array}{c}z^{1} \\ \vdots \\ z^{m}\end{array}\right)$ und $\mathbf{e}=\left(\begin{array}{c}e^{r+1} \\ \vdots \\ e_{m}\end{array}\right)$ die Gleichung

$$
\dot{\mathbf{R}^{\prime} \mathbf{T}^{-1} \mathbf{z}}=\mathbf{e}
$$

so gill auch die Gleichung

. und umgekehrt.

$$
\mathbf{z}^{+}=\mathbf{T}^{+} \mathbf{S}^{-1}\left(\begin{array}{c}
\mathbf{z}^{-} \\
\mathbf{e}
\end{array}\right),
$$


Beweis: a) Man ergänzt (8.4) durch die Identität $\mathbf{T}^{-\mathbf{T}^{-1} \mathbf{z}}=\mathbf{z}^{-}$zu einem quadratischen Gleichungssystem, aus dem dann nach der Auflösung nach z (8.5) folgt.

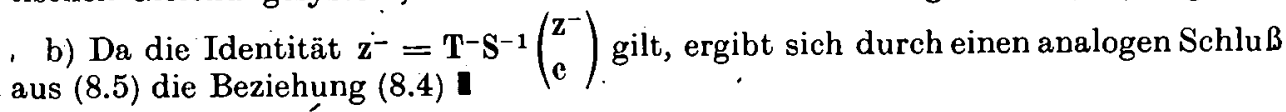

Satz $6:$ Sei $x=0$ und $\mathbf{u} \in C_{m}^{1}\left(\mathfrak{B}^{r}\right)$. Gilt (8.1) sowie $|\mathbf{u}(0, y)-\mathrm{g}(0)| \leqq \varrho$ für $y \in[0, \tau \lambda]$ $\left(\tau \leqq \frac{\sigma}{\lambda}\right)$, so sind auf $[0, \tau \lambda]$ die Randbedingungen (4.2) für $\mathrm{u}$ äquivalent zu den Randbedingungen für $\tilde{\mathbf{u}}$ ( mit $\mathbf{M}=\mathbf{S}^{-1}$ ).

$$
\mathbf{v}^{+}=\left(\mathbf{D}^{+}\right)^{-1}\left(\overline{\mathbf{F}}^{+}-\mathbf{T}^{+} \mathbf{M}\left(\begin{array}{c}
\overline{\mathbf{F}}^{-}-\mathbf{D}^{-} \mathbf{v}^{-} \\
\mathbf{H}
\end{array}\right)\right)
$$

- Beweis: Für alle $y \in[0, \tau \hat{\lambda}]$ ist (4.2) äquivalent zu (8.2). Diese Relation ist wegen $\mathbf{N}=\mathbf{T}^{-1}$ einè Gleichung vom Typ (8.4) mit $\mathbf{u}=\mathbf{u}(0, y), \mathbf{z}=\overline{\mathbf{F}}(\mathbf{0}, y, \mathbf{u}(0, y))-$ $\mathbf{D}(0, y, \mathbf{u}(0, y)) \mathbf{v}(0, y)$. und $\mathbf{e}=\mathbf{H}(y, \mathbf{u}(0, y))$. Hilfssatz 4 liefert die Äquivalenz von (8.2) $\operatorname{mit} \cdot \overline{\mathbf{F}}^{+}-\mathbf{D}^{+} \mathbf{v}^{+}=\mathbf{T}^{+} \mathbf{M}\left(\begin{array}{c}\overline{\mathbf{F}}-\frac{1}{-} \mathbf{D}^{-} \mathbf{v}^{-} \\ \mathbf{H}\end{array}\right)$ bzw. mit (8.6) (wegen (3.4))

Bemerkung 10: Fübren wir eine analoge Einteilung von $2 m$-dimensionalen Vektoren bezüglich der Vorzeichen der Diagonaleleneute der Diagonalmatrix des Systems (6.9) durch, so wird $\tilde{\mathbf{u}}^{-}=\left(\begin{array}{c}\mathbf{u} \\ \mathbf{v}^{-}\end{array}\right)$und $\tilde{\mathbf{u}}^{+}=\mathbf{v}^{+}$. (8.6) nimmit dann die Gestalt $\tilde{\mathbf{u}}^{-}(0, y)=\tilde{\mathbf{H}}\left(y, \tilde{\mathbf{u}}^{-}(0, y)\right)$ für $y \in[0, \tau \hat{\lambda}]$ an mit entsprechend gewähltem $\tilde{\mathbf{H}}$.

Ist V I erfüllt, so haben wir wegen der Konvexität von $\left.\bar{Q}^{0}{ }^{8}\right) S \in C_{m \times m}^{k-1,1}\left(\bar{Q}^{0}\right)$ und. $\widetilde{\mathbf{H}} \in C_{m-\mathbf{r}}^{k-1,1}\left(\bar{Q}^{0} \times\left[-\varrho_{3}, \varrho_{3}\right]^{\top}\right)\left(\varrho_{3}>0\right.$ beliebig $)$.

Es entsteht jetzt die Frage, wie die Kompatibilitätşbedingungen zum Ausgangsproblem P I mit den Kompatibilitätsbedinǵungen $z_{i, 1}$ den abgeleiteten Relationen (6.9), (7.6), (8.6) zusammenhängen. Dazu dienen die beiden folgenden Sätze. Wir kommen dabei ohne Rückgriff auf die Lösungen von (3.5) und (6.9) aus, so daß die, hergeleiteten Zusammenhänge gelten, ohne die Lösbarkeit der entsprechenden Anfangs-Randwertprobleme voraușzusetzen.

Satz 7: Unter $\mathbf{V}$ I sind $z u \mathbf{P} \mathbf{I}$ die Kompatibilitätsbedingungen erster Ordnung (5.1) genuu dann erfüllt, wenn für (6.9), (7.6), (8.6) die Kompatibilitütsbedingungen nullter Ordnung

$$
\mathbf{T}_{10}^{+} \mathbf{g}^{\prime}(0)=\left(\mathbf{D}_{10}^{+}\right)^{-1}\left(\widetilde{\mathbf{F}}_{10}^{+}-\left.\left(\mathbf{T}^{+} \mathbf{M}\right)\right|_{0}\left(\begin{array}{c}
\dot{\mathbf{F}}_{10}^{-}-\left(\mathbf{D}^{-\mathbf{T}^{-}}\right)_{10} \mathbf{g}^{\prime}(0) \\
\mathbf{H}_{10}
\end{array}\right)\right)
$$

erfüllt sind (s. (5.8) und Teil b) des Beweises von Satz 2).

Beweis: (5.1) ist wieder eine Gleichung vom Typ (8.4) mit $\dot{y}=0, \mathbf{u}=\mathbf{g}(0)$ und speziellen Werten $z=\overline{\mathbf{F}}_{10}-\left(\mathbf{D T}^{\prime}\right)_{10} \mathbf{g}^{\prime}(0)$ und $\mathbf{e}=\mathbf{H}_{10}$. Nach Hilfssatz 4 gilt (5.1) génau dann, wenn (8.7), erfüllt ist

Für höhere Regularitätseigenschaften einer Lösung von $\mathbf{P} \mathbf{I}$ ist noch folgender Satz von Wichtigkeit, der Satz 7 ergänzt. Sein Beweis erfolgt durch elementare Umformungen und entsprechende Anwendungen von Hilfssatz 4 unter Berücksichtigung der Gestalt von $\overline{\mathrm{F}}^{*} \cdot[7:$ S. $67-69]$.

8) Vergleiche Fußnote ${ }^{4}$ ) auf Seite 73. 
Satz 8. Unter V I sind zu P I die Kompatibilitütsbedingúngen erster bis.k-ter Ordnung genau dann erfüllt, wenn für (6.9), (7.6), (8.6) die Kompatibilitïtsbedingungen nullter bis $\left(k^{1}-1\right)$-ter Ordnung erfüllt sind.

\section{Die zu P I äquivalente Anfangs-Randwertaufgabe für das Diagonalsystem}

Als Hauptergebnis der bisherigen Úbèrlegungen (Sätze 4 bis 6) können wir jetzt ein Goursat:Problem $\mathbf{P}$ II für das verlängerte System (6.9) aufstellen, däs dem Ausgangsproblem $\mathbf{P}$ I in $\mathfrak{B}^{\mathrm{r}}$ äquivalent ist in dem Sinne, daß jeder Lösung des einen Problems eine Lösung des anderen entspricht.

PIII: Gesucht wird eine Lösung $\left(\begin{array}{l}\mathbf{u} \\ \mathbf{v}\end{array}\right)$ von (6.9) in $\mathfrak{B}^{2}$, die die Anfangswérte (7.6) auf $[0,1]$ der $x$-Achse annimmt und die Randbedingungen (8.6) auf $[0, \tau i]$ der $y$-Achse erfüllt.

Bemerkung 11: Entsprechend der Voraussetzungen für die oben genannten Sätze ist es notwendig, neben einer Lösung von $\mathbf{P} \mathbf{I}$ bzw. P Il ein $\tau(0<\tau$ $\left.\leqq \min \left\{1, \frac{\sigma}{\lambda}\right\}\right)$ so bestimmen zu können, daß $\|\mathbf{u}-\mathbf{g}\|_{\mathfrak{B} r} \leqq \varrho$ gilt. Das ist auch bei den folgenden Betrachtungen vorauszusetzen.

Wir werden uns bei dem Existenzbeweis [8] mit dem Goursat-Problem P IlI für cin belicbiges Diagonalsystem befassen, dessen Lösbarkeit die Auflösung von Problem P II und damit von P I beinhaltet.

P III : Bestimme $\mathbf{u} \in C_{m}^{0.1}\left(\mathfrak{B}^{r}\right)$ als Lösung̈ von

$$
\frac{\partial \mathbf{u}}{\partial y}+\mathbf{D}(x, y, \mathbf{u}) \frac{\partial \mathbf{u}}{\partial x}=\mathbf{F}(x, y, \mathbf{u}) \quad \text { t. } \ddot{u} \text {. in } \dot{\mathfrak{B}} \mathbf{r}
$$

mit $\mathbf{u}(x, 0)=\mathbf{g}(x)$ für $x \in[0,1]$

.und $\quad \mathbf{u}^{+}(0, y)=\mathbf{H}\left(y, \mathbf{u}^{-}(0, y)\right)$. für $y \in[0, \tau \hat{\lambda}]$.

Wir stellen die folgenden Voraussetzungen.

V III : $\mathbf{D} \in C_{m \times m}^{0.1}(\bar{Q}), \mathbf{F} \in C_{m}^{0.1}(\bar{Q}), g \in C_{m}^{0.1}([0,1]), \mathbf{H} \in C_{m-r}^{0.1}\left(\bar{Q}^{-}\right)$mit

$\bar{Q}^{-}=\left\{\left(y, u^{1}, \ldots, u^{r}\right) \in \mathbf{R}^{1+r},|0 \leqq y \leqq \sigma,| \mathbf{u}^{-}-\mathrm{g}(0) \mid \leqq \varrho\right\}$

In $\bar{Q}$ gelte für die Diagonalelementé $D^{i}$ von $\mathbf{D}$ wiederum die Anỏrdinung (3.4). Für (4.1) und (9.2) seien die Kompatibilitätsbedingungen' nullter Ordnung

$\mathbf{g}^{+}(0)=\mathbf{H}\left(0, \mathbf{g}^{-}(0)\right)$

erfiillt.

Um das Existenzresultat für P III auf das Ausgangsproblem P I übertragen zu können, sind die Åquivalenzsätze über die Kompatibilitätsbedingungen (Sätze : 7 und 8) von Wichtigkeit. Wir kommen damit nämlich zu den folgenden Schlußfolgerungen.

Satz 9: Sei P III unter den Bedingungen V III lösbar. Gelten V I und. (5.1), so existiert eine Lösung $\mathbf{u} \in C_{m}^{1,1}\left(\mathfrak{B}^{\top}\right)$ von $\mathbf{P} \mathbf{I}$. 
Bewe is: Nach Sat 7 folgt aus der Gültigkeit von (5.1) das Erfülltsein der Kompatibilitätsbedingungen nullter Ordnung (8.7) zu P II. Damit ist P II ein GoursatProblem vom Typ PIII mit $2 m$ Differentialgleichungen und speziell gewählten D, F, $\mathbf{H}$ und g (vgl. auch Satz 4, Bemerkung 10), welche die entsprechenden. Voraussetzungen V III erfüllen. Folglich existiert zu P II eine Lösung $\left(\begin{array}{l}\mathbf{u} \\ \mathbf{v}\end{array}\right) \in C_{2 m}^{0.1}\left(\mathfrak{B}^{\mathrm{v}}\right)$. Anwendung von Satz 5 für $k=1$ ergibt die Behauptung, wobei die Erfüllung der Randbedingungen (4.2) aus Satz 6 folgt

Es ist nun bemerkenswert, daß bereits die Lösbarkeit von P III und unsere bisherigen Überlegungen ausreichen, um zum Ausgangsproblem P I die Existenz einer Lösung mit höheren Regularitätseigenschaften nachzuweisen.

Folgerung: Sei P III'unter den Bedingungen.V III lösbur. Unter der Gültigkeit von V I und der Kompatibilitütsbedingungen erster bis k-ter Ordnung besitzt $\mathbf{P} \mathbf{I}$ eine Lösung $\mathbf{u} \in C_{m}^{k, 1}\left(\mathfrak{Q Z}^{\mathrm{r}}\right)$.

Beweis (durch Induktion über $k \geqq 1 ; k=1$ : Satz 9): Sei.obige Aussage richtig für $k=\varkappa$. Sind nun V I mit $k=x+1$ sowie die Kompatibilitätsbedingungen erster bis $(x+1)$-ter Ordnung zu P I erfiillt, so ergibt Satz 8 die Gültigkeit derjenigen zu P II von nullter bis $x$-ter Ordnung. P II wird jetzt als spezielles Goursat-Problem vom Typ $\mathbf{P} \mathbf{I}^{9}$ ), betrachtet, wobei auch die zugehörigen Voraussetzungen $\mathbf{I}$ mit $k=*$ gelten (s. Satz 4, Bemerkung 10). Dann liefert die Induktionsvoraussetzung einé Lösung $\left(\begin{array}{l}\dot{u} \\ v\end{array}\right)$ von P II aus der Klasse $C_{2 m}^{x, 1}\left(\mathfrak{B}^{r}\right)$. Aus Satz 5 mit $k=x+1$ sowie Satz 6 folgt wie im Beweis des vorangehenden Satzes, da $\$$ u eine Lösung von P I aus $C_{m}^{x+1.1}\left(\mathfrak{B}^{x}\right)$ ist

Mit diesen beiden Sätzen ist die Lösbarkeit unseres Ausgangsproblems P I vollständig auf die Lösbarkeit des speziellen Goursat-Problemıs P III reduziert worden, bei den sich auf Grund der Diagonalform des quasilinearen hyperbolischen Systems (9.1) und der expliziten Randrelationen (9.2) das Differenzenverfahren relativ einfach gestalten wird. Außerdem braucht nur die $L$-Sțetigkeit einer Iösung von P III nachgewiesen zu werden.

\section{ILITERATUR}

[1] Вескект, Н.: Uber quasilineare hyperbolische Systeme partieller Differentialgleichungen erster Ordnung mit zwei unabhängigen Variablen. Das Anfangswertproblem, die gemischte. Anfangs.Randwertaufgabe, das charakteristische Problem. Ber. Sächs. Akad. Wiss;, Math.-Nat. KI. 97 (1950), $3-68$.

[2] Beскент, H.: Bemerkungen über die Verbiegüng hyperbolisch gekrümmiter Flächenstücke. Ber. Sächs. Akad. Wiss., Math.-Nat. KI. 93 (1951), 3-15.

[3] Courant, R., and K. O. Friedricus: Supersonic flow and shock waves. New York: Interscience Publishers 1948 .

[4] Covrant, R., and D. Hilbert: Methods of mathematical physics. Part II: Partial differential equations. New York-London: Interscience Publishers 1962.

[5] Counast, R., and P. LaX: Nonlinear partial differential equations with two independent 'variables. Comm. Pure Appl. Math. 2 (1949), 255-273.

9) Wegen der Gültigkeit der Kompatibilitätsbedingungen nullter Ordnung zu P II können die Randbedingungen (8.6) auf $[0, \tau \hat{\lambda}]$ äquivalent in die entsprechenden Differentialrelationen vom Typ (4.2) umgeformt werden. 
[6] Gitrel, H.-P.: Zum Goursat.Problem für' quasilineare hyperbolische Systeme partieller Differentialgleichungen erster Ordnung mit zwei unabhängigen Variablen und dessen Anwendung in bodenmechanischen Aufgabenstellungen. Diplomarbeit. Leipzig: Karl-Marx: Universität 1979.

[7] Gitrél, H.-P.: Das Goursat-Problem für quasilineare hýperbolische Systeme partieller Differentialgleichungen erster Ordnung mit zwei unabhängigen Vàriablen. Existenz- und Eindeutigkeitsbeweis für dessen Lösung mittels Differenzenverfahren. Dissertation. Leipzig: Karl-Marx-Universität 1981.

[8] Gitrel, H.-P.: Existenz- und Eindeutigkeitsbeweis zur Lösung des Goursat-Problems für quasilineare hyperbolische Systeme erster Ordnung mittels Differenzenverfahren. $Z$. Anal. Anw. 4 (1985) 6 (erscheint):

[9] Kolmogorov, A. N., und S.V. Foms: Reelle Funktionen und Funktionalanalysis: Ber. lin: Dt. Verlag Wiss. 1975.

[10] KRSYZaxski, M., und J: SCHa uder: Quasilineare Differentialgleichungen zweiter Ordnung vom hyperbolischen Typus. Gemischte Randwertaufgaben. Studia Math. 6 (1936), 162189.

[11] Kufrer, A., Johs, O., and S. Fučrk: Function spaces. Prague: Academia 1977.

[12] Perrox, O.: Úber die Existenz und Nichtexistenz von Integralen partieller Differentialgleichungssysteme im reellen Gebiet. Math. Z. $\mathbf{9 7}(1928), 549-564$.

[13] Prouse, G.: Sulla risoluzione del problema misto perle equazioni iperboliche non lineari mediante le differenze finite. Ann. Mat. Pura Appl. (Serie 4) 46 (1958), 313-343.

[14] RaDemacher, H.: Uber partielle und totale Differenzierbarkeit von Funktionen mehrer Variabler und über die Transformation der Doppelintegrale. Math. Ann. 79 (1919), 340 -359 .

[15] Tromé, V.: Difference methods for two dimensional-mixed. problems for hyperbolic first order systems. Arch. Rational Mech. Anal. S (1961), $69-88$.

[16] Thonée, V.: A mixed boundary-value problem for hyperbolic first order systems with derivatives in the boundary conditions. Arch. Rational Mech. Anal. $S$ (1961), 435-443.

[17] Zejoler, E.: Vorlesungen über nichtlineare Funktionalinalysis. I: Fixpunktsätze. Leipzig: BSB B. G. Teubner Verlagsgesellschaft 1976.

Manuskripteingang: 16.08.1983; in revidierter Fassung: 12.03. 1984

\section{VERFASSER :}

Dr. Hans-Pethi GitTel

- Sektion Mathematik der Karl-Marx-Universität

DDR - 7010 Leipzig, Karl-Marx-Plittz 10 